\title{
Molecular Auger Interferometry
}

\author{
M. A. Khokhlova, ${ }^{1, *}$ B. Cooper, ${ }^{2}$ K. Ueda, ${ }^{3}$ K. C. Prince, ${ }^{4,5}$ P. Kolorenč, ${ }^{6}$ M. Yu. Ivanov, ${ }^{1,7}$ and V. Averbukh ${ }^{1}$ \\ ${ }^{1}$ Blackett Laboratory, Imperial College London, London SW7 2AZ, United Kingdom \\ ${ }^{2}$ Department of Physics and Astronomy, University College London, London WC1E 6BT, United Kingdom \\ ${ }^{3}$ Institute of Multidisciplinary Research for Advanced Materials, Tohoku University, 980-8577 Sendai, Japan \\ ${ }^{4}$ Elettra-Sincrotrone Trieste SCpA, 34149 Basovizza-Trieste, Italy \\ ${ }^{5}$ Molecular Model Discovery Laboratory, Swinburne University of Technology, 3122 Hawthorn, Australia \\ ${ }^{6}$ Charles University, Institute of Theoretical Physics, V Holešovičkách 2, 18000 Prague, Czech Republic \\ ${ }^{7}$ Max Born Institute for Nonlinear Optics and Short Pulse Spectroscopy, Max-Born-Strasse 2A, Berlin D-12489, Germany
}

(Received 26 February 2019; published 11 June 2019)

\begin{abstract}
We introduce and present a theory of interferometric measurement of a normal Auger decay lifetime in molecules. Molecular Auger interferometry is based on the coherent phase control of Auger dynamics in a two-color $(\omega / 2 \omega)$ laser field. We show that, in contrast to atoms, in oriented molecules of certain point groups the relative $\omega / 2 \omega$ phase modulates the total ionization yield. A simple analytical formula is derived for the extraction of the lifetimes of Auger-active states from a molecular Auger interferogram, circumventing the need in either high-resolution or attosecond spectroscopy. We demonstrate the principle of the interferometric Auger lifetime measurement using inner-valence decay in $\mathrm{CH}_{3} \mathrm{~F}$.
\end{abstract}

DOI: $10.1103 /$ PhysRevLett.122.233001

Auger decay $[1,2]$ is a fundamental process, where in the presence of an inner-shell vacancy, a valence electron recombining with this vacancy supplies another (Auger) electron with sufficient energy to leave an atom or a molecule. Auger decay is intensely studied due to its importance for such diverse areas as surface science, atmospheric chemistry, radiation biology, $\mathrm{x}$-ray spectroscopy, and attosecond physics.

Advances in laser and x-ray technology have initiated a new chapter in the study of Auger processes, enabling timeresolved measurements of the Auger decay lifetimes [3], matching the spectral resolution of the best available energy-resolved experiments [4]. Furthermore, several new Auger-based radiative processes have been explored, such as laser-assisted Auger decay (LAAD) [5-7] or laserenabled Auger decay (LEAD) [8] with multiple photons. In both of them, the Auger decay is initiated by the x-ray and laser pulses. For LAAD the Auger decay is energetically allowed, whereas in the case of LEAD, the Auger process is initially energetically forbidden, but becomes allowed if the system can absorb additional energy from a laser field. Another example is single-photon laser-enabled Auger decay (spLEAD), first predicted theoretically in [9]. In this process, the ionization of an Auger-inactive ionic state occurs due to a two-electron transition filling the inner-valence vacancy with simultaneous photon absorption. Recently, atomic spLEAD was confirmed experimentally [10] using an $\omega / 2 \omega$ coherent control scheme [11] and light from a freeelectron laser (FEL). Variation of the relative phase of the two frequencies resulted in modulation of the angular distribution of the electrons emitted by ionized $\mathrm{Ne}$ atoms.
We show analytically that for oriented molecules belonging to the molecular point groups $C_{s}, C_{n}, C_{n v}, C_{\infty v}, C_{3 h}$, $D_{3}, D_{3 h}$, and $T_{d}$, one can control not merely the angular distribution but also the total yield of the Auger or spLEAD electrons or, equivalently, the yield of doubly ionized molecules. Molecular orientation in the gas phase has been repeatedly demonstrated [12]. It is well recognized that the total yield can be controlled even in the atomic case via schemes employing same-parity transitions, e.g., $\omega / 3 \omega$; however within the photon energy regime relevant for the Auger dynamics practical realization of such control schemes appears problematic [13].

Here, we present Auger interferometry, i.e., a theory of coherent control of Auger decay and spLEAD in ionized molecules. The theory is applied to the $\mathrm{CH}_{3} \mathrm{~F}$ ion which has an Auger-active state (AAS) with energy only slightly above the double ionization potential (DIP) and, as a result, a decay width that is challenging to predict theoretically. We show that attosecond resolution can be gained without using attosecond pulses, by simply controlling the relative $\omega / 2 \omega$ phase. The accuracy of the retrieved time-resolved dynamics is determined by the accuracy with which the $\omega / 2 \omega$ phase difference is controlled, and by the accuracy with which intensities of the $\omega$ field and $2 \omega$ field and transition dipole moments are determined.

We study the ionization dynamics of a molecule under the influence of two long XUV (FEL) pulses. In general, the system can have several AASs within the energy region around the resonant energy. We consider the ground state $\varphi_{0}^{N-1},|0\rangle, M$ AASs $\varphi_{m}^{N-1},|m\rangle,(m=1, \ldots, M)$ with $N-1$ electrons, and a doubly ionized state $|E\rangle$, described by the 
electron in the continuum $\chi_{E}^{n}$ and the associated dicationic state $\varphi_{n}^{N-2}$, where $n$ is the index of the ionic channel. The AASs $|m\rangle$ are above the DIP. In the case of spLEAD the states $|m\rangle$ are below the DIP.

The laser field transfers the molecule from the ground state $|0\rangle$ to the doubly ionized state $|E\rangle$ by two interfering ionization paths, directly by $2 \omega$-photon absorption, and by absorption of two $\omega$ photons via the intermediate decaying states $|m\rangle$. The faster the intermediate states decay, the weaker the second path becomes, so that the maximum yield of emitted electrons is limited by the lifetimes of the $|m\rangle$ states. Destructive interference or complete suppression of the yield requires a balance of the paths that can be reached by tuning the intensity of the $\omega$ field relatively to the intensity of the $2 \omega$ field.

In molecules, this interference between single- and twophoton transitions (a resonant multiphoton ionization process [14]) is possible if the expansion of the direct product of the representation of a dipole operator or components thereof, $D_{\text {dipole, }}$ itself contains the dipole representation:

$$
D_{\text {dipole }} \otimes D_{\text {dipole }} \supset D_{\text {dipole }}
$$

This is for the present case of linear, parallel polarization of the two frequencies which implies that the dipole operator components belong to the same irreducible representation (IRREP). The case of orthogonal polarizations is considered in the Supplemental Material (SM) [15] and the allowed point groups for linear polarization with perpendicular polarization of $\omega$ and $2 \omega$ are listed. The condition (1) is trivially fulfilled for all symmetry groups where one of the dipole components belongs to the fully symmetric IRREP, namely for $C_{s}, C_{n}, C_{n v}$, and $C_{\infty v}$. Apart from those symmetry groups, the condition (1) is satisfied for molecules of the point group symmetries $D_{3}, D_{3 h}$, and $T_{d}$.

To describe theoretically the molecular Auger interferometry, we solve the time-dependent Schrödinger equation (TDSE), atomic units (a.u.) are used throughout,

$$
i \frac{\partial \Psi^{N-1}(t)}{\partial t}=\hat{H}(t) \Psi^{N-1}(t)
$$

for the Hamiltonian $\hat{H}(t)=\hat{H}_{0}+\hat{V}(t)$, where $\hat{H}_{0}$ is the unperturbed Hamiltonian of the $(N-1)$-electron system

$$
\begin{aligned}
\hat{H}_{0} \varphi_{m}^{N-1} & =\tilde{E}_{m}^{N-1} \varphi_{m}^{N-1}, \\
\hat{H}_{0}\left[\chi_{E}^{n} \varphi_{n}^{N-2}\right] & =E_{n}^{N-1}\left[\chi_{E}^{n} \varphi_{n}^{N-2}\right] .
\end{aligned}
$$

The energies $\tilde{E}_{m}^{N-1}=E_{m}^{N-1}-i \Gamma_{m}$ of the AASs $|m\rangle$ $(m=1, \ldots, M)$ take into account the natural energy (or decay) half width at half maximum (HWHM) $\Gamma_{m}$, denoted below "the energy width." For the ground state $|0\rangle$ the energy is real $\tilde{E}_{0}^{N-1}=E_{0}^{N-1}$, and $E_{n}^{N-1}=E_{n}^{N-2}+E$ is the energy of the doubly ionized state, with $E$ the energy of the continuum electron. The term $\hat{V}(t)=-d \mathcal{E}$ describes the interaction with a linearly polarized two-color laser field

$$
\mathcal{E}=\mathcal{E}_{1} \cos (\omega t)+\mathcal{E}_{2} \cos (2 \omega t+\phi),
$$

where $\mathcal{E}_{1}$ and $\mathcal{E}_{2}$ are the electric field amplitudes of the field with frequencies $\omega$ and $2 \omega$, respectively, and $\phi$ is their relative phase.

The total wave function $\Psi^{N-1}(t)$ is given by

$$
\begin{aligned}
\Psi^{N-1}(t)= & c_{0}(t) \varphi_{0}^{N-1} e^{-i E_{0}^{N-1} t}+\sum_{m=1}^{M} c_{m}(t) \varphi_{m}^{N-1} e^{-i \tilde{E}_{m}^{N-1} t} \\
& +\sum_{n} \int d E c_{E}^{n}(t) \chi_{E}^{n} \varphi_{n}^{N-2} e^{-i E_{n}^{N-1} t}
\end{aligned}
$$

The last term in Eq. (5) includes a sum over different ionic channels $n$.

We substitute the total wave function (5) into the TDSE (2), and by using the standard rotating-wave approximation, obtain the usual system of differential equations for the complex amplitudes [see (S2) in the SM]. This system of equations for the induced transitions between the ground state of the ionized molecular system, single AAS $(M=1)$, and the doubly ionized state of the molecule with an electron in the continuum (see Fig. 1), can be solved fully analytically [neglecting field-induced transitions between doubly ionized states described by the transition dipole moments $d^{n n^{\prime}}$ (S3) which are much weaker than the other transitions in our case] by applying the procedure of the

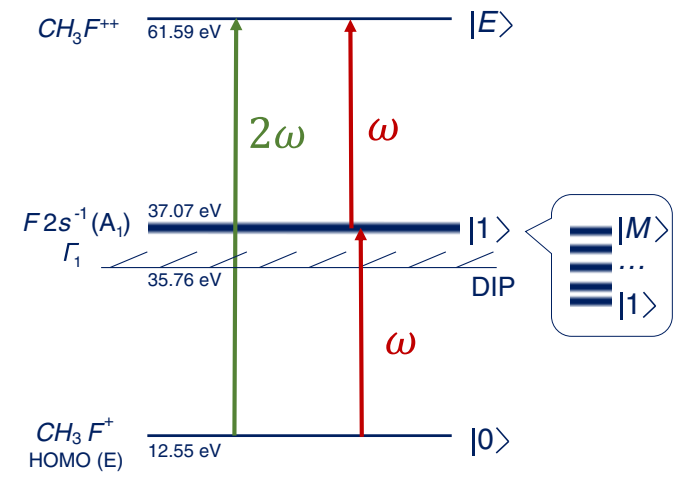

FIG. 1. Auger interferometry scheme for the case of the coherent control of Auger decay of a F $\left(2 \mathrm{~s}^{-1}\right)$ hole in $\mathrm{CH}_{3} \mathrm{~F}$ with $\omega$ and $2 \omega$ laser fields. The frequency of the transition between the ground state $|0\rangle=\varphi_{0}^{N-1}$ of the molecular cation and the AAS $|1\rangle=\varphi_{m}^{N-1}$ (or a series of them) with energy HWHM $\Gamma_{1}$ equals the fundamental frequency $\omega$. The doubly ionized final state $|E\rangle=\chi_{E}^{n} \varphi_{n}^{N-2}$ can be reached by two paths: either from the ground state directly by absorbing a $2 \omega$ photon or from the ground state via the intermediate AAS by absorbing two $\omega$ photons. Interference between these two transitions can be controlled by the relative phase $\phi$ between the two laser fields. 
adiabatic elimination of the continuum [25-27]. The case of two AASs is discussed separately in the SM. The complex amplitudes of the continuum states become stationary when the XUV pulse is much longer than the lifetimes of the considered states, which formally corresponds to taking the $t \rightarrow \infty$ limit. In this case the full timedependent expression for $c_{E}^{n}$ can be simplified:

$$
c_{E}^{n}=-\frac{\alpha^{n}}{(\tilde{E}-i \tilde{\Gamma}-E)^{2}-\delta^{2}},
$$

where $\alpha^{n}$ depends on the molecular parameters including $\Gamma_{1}$, and $\delta$ can be associated with the doubled complex Rabi frequency [28] (see the SM for more detail). Here, $\tilde{E}=$ $\frac{1}{2}\left(E_{0}+E_{1}+3 \omega\right)$ is a manifestation of the energy conservation law and $\tilde{\Gamma}=\frac{1}{2}\left(\Gamma_{0}^{i}+\Gamma_{1}^{i}+\Gamma_{1}\right)$ is a total energy HWHM, where $\Gamma_{0}^{i}=2 \pi \sum_{n}\left|V_{0 E}^{n}\right|^{2}$ and $\Gamma_{1}^{i}=2 \pi \sum_{n}\left|V_{1 E}^{n}\right|^{2}$ are the ionization widths of the ground $|0\rangle$ and the intermediate $|1\rangle$ states, respectively.

Generally, Eq. (6) looks similar to a Lorentzian profile but has a much more complicated character due to the complex form of $\delta$, and is less amenable to straightforward analysis. However, for the resonant case $\left(\Delta_{01}=E_{1}-E_{0}-\omega=0\right)$ Eq. (6) can be rewritten in the form allowing analysis. In this case the resonant yield can be presented in the form

$$
\left|c_{E}^{r}\right|^{2}=\frac{V_{01}^{2}\left|V_{1 E}\right|^{2}+\left|V_{0 E}\right|^{2} \Gamma_{1}^{2}+2 V_{01}\left|V_{0 E}\right|\left|V_{E 1}\right| \Gamma_{1} \sin \left(\phi+\phi_{01}\right)}{\left(V_{01}^{2}+\Gamma_{1} \Gamma_{0}^{i}\right)^{2}+\left[4 \pi V_{01}\left|V_{1 E}\right|\left|V_{0 E}\right| \cos \left(\phi+\phi_{01}\right)\right]^{2}},
$$

where the matrix elements $V_{01}=-d_{01} \mathcal{E}_{1} / 2, \quad V_{0 E}=$ $-d_{0 E} \mathcal{E}_{2} / 2$, and $V_{1 E}=-d_{1 E} \mathcal{E}_{1} / 2$ contain transition dipoles $d_{01}, d_{0 E}=\sum_{n}\left|d_{0 E}^{n}\right| e^{i \phi_{0}^{n}}$, and $d_{1 E}=\sum_{n}\left|d_{1 E}^{n}\right| e^{i \phi_{1}^{n}}$ [see (S3) in the SM]. Equation (7) is derived within the assumption that the phase difference $\phi_{01}^{n}=\phi_{0}^{n}-\phi_{1}^{n}$ is constant for different channels $\left(\phi_{01}^{n} \approx \phi_{01}\right)$ of a particular molecular system (see SM for details). The dependence of the resonant yield (7) on the relative phase $\phi$ is contained in the numerator and disappears when $\Gamma_{1}=0$. In the denominator the dependence on $\phi$ is weak and can be neglected if at least one of the following conditions is satisfied:

$$
\begin{aligned}
\mathcal{E}_{1}^{2} / \mathcal{E}_{2} & \ll \Gamma_{1}\left|d_{0 E}\right| /\left(2 d_{01}\left|d_{1 E}\right|\right), \\
\mathcal{E}_{2} & \ll d_{01} /\left(2 \pi\left|d_{0 E}\right|\left|d_{1 E}\right|\right) .
\end{aligned}
$$

The conditions (8) correspond to the regime of moderate intensities, when only processes described by the first order of perturbation theory contribute sufficiently. In this case, the resonant yield (7) is modulated and the modulation depth $\mathcal{M}$ can be derived as

$$
\mathcal{M}=2 /\left(\frac{V_{01}\left|V_{1 E}\right|}{\left|V_{0 E}\right| \Gamma_{1}}+\frac{\left|V_{0 E}\right| \Gamma_{1}}{V_{01}\left|V_{1 E}\right|}\right)
$$

for given $\Gamma_{1}$. The modulation depth (9) maximum value is 1 and can be achieved when $\left|V_{0 E}\right| \Gamma_{1}=V_{01}\left|V_{1 E}\right|$. This means that for any value of $\Gamma_{1}$ a high contrast $\mathcal{M}$ can be obtained by tuning the field intensities. The modulation depth $\mathcal{M}$ can be measured experimentally within the relative phase scan of the resonant yield and used for extraction of the AAS energy width $\Gamma_{1}$ value within a simple analytical expression

$$
\Gamma_{1}=\frac{1-\sqrt{1-\mathcal{M}^{2}}}{\mathcal{M}} \frac{V_{01}\left|V_{1 E}\right|}{\left|V_{0 E}\right|} .
$$

Here, one can notice that $\Gamma_{1}$ is proportional to the $\omega$-field intensity $I_{1}$ and inversely proportional to the square root of the intensity $I_{2}$ of the $2 \omega$ field $\left(I_{1,2}=\mathcal{E}_{1,2}^{2}\right)$. Transition dipole moments can be calculated using, for instance, the algebraic diagrammatic construction (ADC) technique [29]. The other way to find the factor $d_{01}\left|d_{1 E}\right| /\left|d_{0 E}\right|$ is to carry out two additional measurements: (i) with only $2 \omega$ field giving the absolute value of the transition dipole moment $d_{0 E}$ which can be extracted from the yield $\left|c_{E}^{r}\right|^{2}=1 /\left(4 \pi^{2}\left|d_{0 E}\right|^{2} I_{2}\right)$, and (ii) with only $\omega$ field giving the yield $\left|c_{E}^{r}\right|^{2}=\left|d_{1 E} / d_{01}\right|^{2}$ from which the transition dipole moment $d_{1 E}$ absolute value can be found by using the transition dipole moment $d_{01}$, the value of which is relatively easy to calculate with the accuracy of $5 \%-10 \%$ or better [30-32].

To demonstrate the principal of the molecular Auger interferometry, we choose a molecular system, fluoromethane $\left(\mathrm{CH}_{3} \mathrm{~F}\right)$, that has a $2 s$-ionized AAS [33-36] with an energy only slightly above the DIP. We study the dynamics of the oriented singly ionized molecule $\mathrm{CH}_{3} \mathrm{~F}$ calculating the behavior of the total yield $\int d E\left|c_{E}\right|^{2}$, see Eq. (6) either of doubly ionized molecules or electrons. We calculate the transition dipole moments using the ab initio many-body Green's function-based technique called ADC [29], of extended second order, $\operatorname{ADC}(2) x$. Computational details are discussed in the SM.

We consider the parameters of the field (4) such as the intensity of each field component and the frequency $\omega$ which changes with the detuning from the resonance $\Delta_{01}$. First, we investigate the behavior of the line shape of the emitted electrons $\left|c_{E}\right|^{2}$. The line shape as a function of the relative phase $\phi$ for selected intensities shown in Fig. 2 demonstrates that for all values of the relative phase $\phi$ we observe an asymmetry of the spectrum relative to the central energy $E=E_{0}+2 \omega$ of the emitted electrons. The emission is on average stronger for the relative phase 


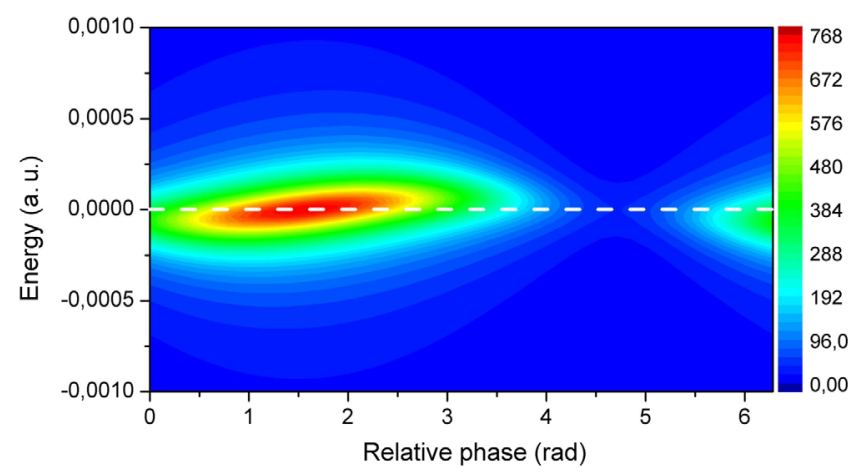

FIG. 2. The spectrum of emitted electrons ionized by a laser field (4) for the case of $\mathrm{CH}_{3} \mathrm{~F}^{+}$with the energy width $\Gamma_{1}$ as a function of the relative phase $\phi$. The zero of the electron energy $E$ ( $y$ axis) corresponds to the resonant energy $E_{0}+2 \omega$. The intensities of the $\omega$ field and $2 \omega$ field are $6.7 \times 10^{12} \mathrm{~W} / \mathrm{cm}^{2}$ and $\Gamma_{1}=5 \times 10^{-3}$ a.u..

$\phi$ in the interval $0-\pi$, while for the $\pi-2 \pi$ region it is weaker or has disappeared ("switched off"). Detailed results are given in the SM. This can be a basis for phase control of the emitted electron spectrum by setting the relative phase $\phi$ for appropriate intensities. Since the value of $\Gamma_{1}$ is unknown, it was chosen to lie in within the range of $10^{-3}-10^{-2}$ a.u.
We show that the AAS energy width value can be retrieved from the total yield of the emitted electrons $\left(\int d E\left|c_{E}\right|^{2}\right)$, and this yield is controlled by the relative phase $\phi$ and intensities $I_{1,2}$. Figures 3(a)-3(c) present Auger interferograms showing the dependence of the population of the doubly ionized state of $\mathrm{CH}_{3} \mathrm{~F}$ (or the electron yield) as a function of the relative phase $\phi$ and the energy width $\Gamma_{1}$ of the singly ionized AAS of $\mathrm{CH}_{3} \mathrm{~F}$, for the different intensity ratios. One can see that for the different decay widths, the depth of the population modulations varies. In Fig. 3(d) we show the dependence of the modulation depth on the energy width $\Gamma_{1}$ summarizing the results presented in Auger interferograms in Figs. 3(a)-3(c) as well as the detuned case discussed in the SM. Here, one can see that the detuning from the resonance $\Delta_{01}$ affects the modulation depth of the total yield. Also one can notice that the intensity ratio equal to 1 corresponds to a higher modulation depth on average over the whole range of $\Gamma_{1}$ values. Figure 3(d) shows the comparison between the total and resonant yield (9) for zero detuning (see the SM for more details) which shows an excellent agreement when the conditions (8) are satisfied. This provides a way to extract the AAS energy width using the simple expression (10). For the case when two different values of $\Gamma_{1}$ correspond to one value of

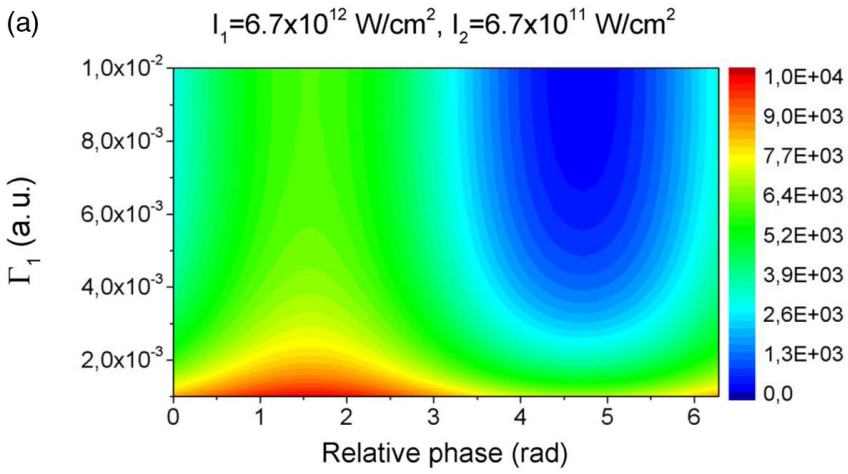

(b) $I_{1}=6.7 \times 10^{12} \mathrm{~W} / \mathrm{cm}^{2}, \mathrm{I}_{2}=6.7 \times 10^{12} \mathrm{~W} / \mathrm{cm}^{2}$
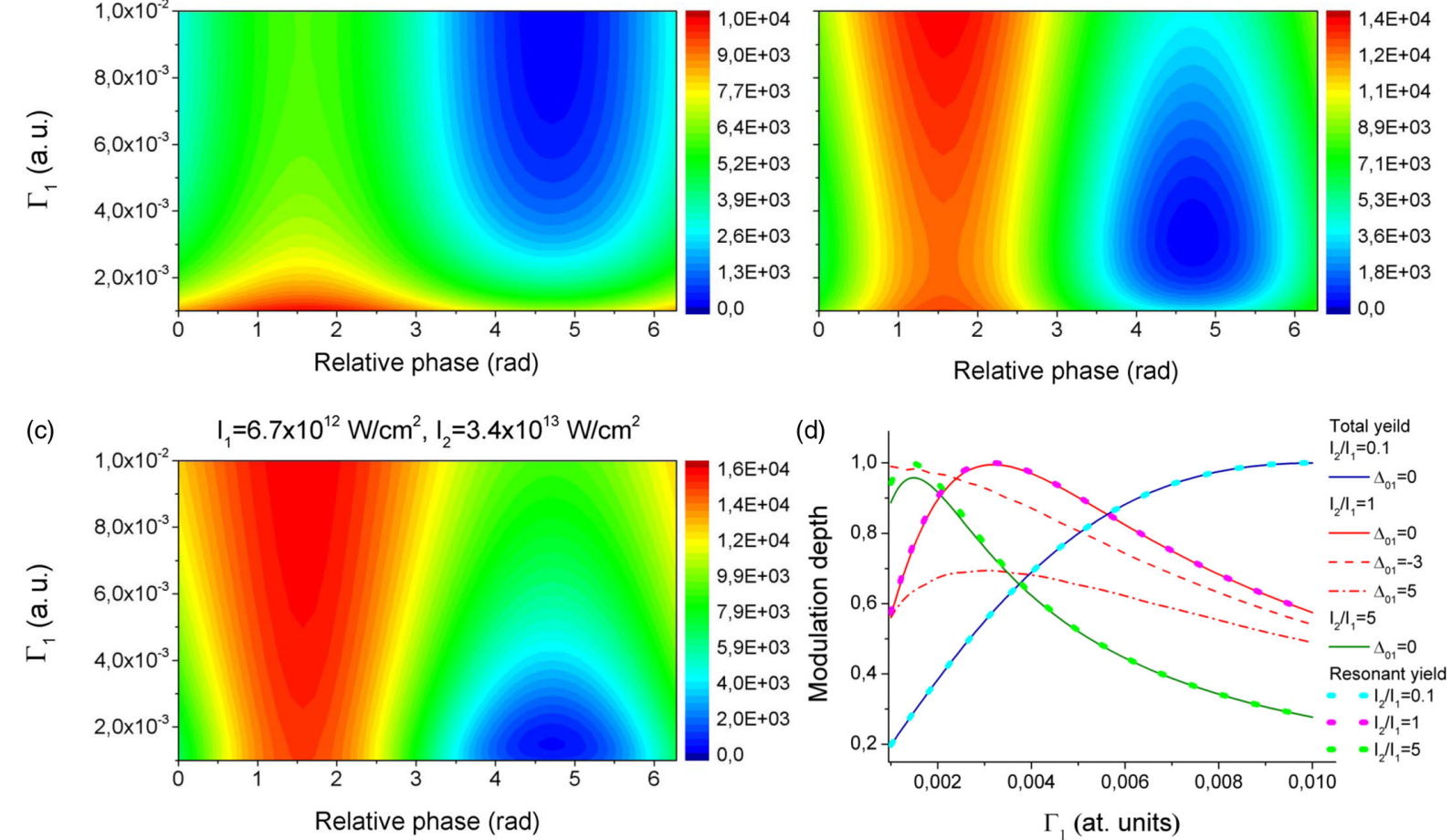

FIG. 3. (a)-(c) Auger interferograms. The total yield of electrons emitted by a bichromatic laser field for the $\mathrm{case}$ of $\mathrm{CH}_{3} \mathrm{~F}^{+}$as a function of the relative phase $\phi$ and the $\Gamma_{1}$ ( $y$ axis). (d) Modulation depth of the total yield for zero (thin solid lines) and nonzero detuning (thin dashed lines), and the resonant yield (thick dotted lines) as a function of the AAS energy width $\Gamma_{1}$. The intensities are the same as for (a)-(c) used, and detunings $\Delta_{01}$ are shown in $10^{-3}$ a.u. 
modulation depth $\mathcal{M}$ [see Fig. 3(d)] the uncertainty can be eliminated by carrying out one additional measurement with changed intensity ratio. For instance, if the $\omega$ field is kept constant and the intensity ratio is reduced by decreasing the $2 \omega$-field intensity, then if the modulation depth becomes higher it corresponds to the higher value of the energy width and vice versa. This illustrates that information about an AAS such as energy width (or lifetime) can be obtained using this interferometric method.

In summary, we introduced molecular Auger interferometry as a measurement of the total yield of a normal Auger decay (or spLEAD) in molecules of particular point groups as a function of the relative $\omega / 2 \omega$ phase. Our analytical theory predicts that the Auger decay lifetime can be reconstructed from the relative phase scan of the Auger yield modulation. The interference contrast onto which the decay width is mapped [Eqs. (9) and (10)] can be maximized at any decay width by controlling the ratio of the $\omega$ - and $2 \omega$-field intensities. As a result, the interferometric measurement proposed here is free of the limitations of both high-resolution Auger electron spectroscopy (struggling to characterize decay widths smaller than the instrumental energy resolution) and attosecond time-resolved spectroscopy (struggling to characterize decay rates faster than the available pulse durations). We suggest a method of extracting this information from the Auger interferograms by applying a simple analytical formula. An illustrative example dealing with inner-valence hole decay in $\mathrm{CH}_{3} \mathrm{~F}$ shows that molecular Auger interferometry is well within the present-day experimental capabilities of the modern FEL facilities, such as FERMI@Elettra. More generally, the same coherent control scheme can be used to study any type of ultrafast hole dynamics, e.g., hole migration $[37,38]$.

M. K. acknowledges support by The Royal Society through Newton International Fellowship (NF161013). B.C. acknowledges financial support STFC ST/ R005133ST/1. K. U. acknowledges support by "Five-star Alliance" in "NJRC Mater. \& Dev." and the IMRAM project. P. K. acknowledges financial support by the Czech Science Foundation (Project GAČR No. 1710866S). V. A. and M. I. acknowledge support by EPSRC/DSTL MURI-MIR Grant No. EP/II08680/1.

*m.khokhlova@imperial.ac.uk

[1] L. Meitner, Z. Phys. 9, 131 (1922).

[2] P. Auger, Compt. Rendus. Acad. Sci. 177, 169 (1923).

[3] M. Drescher, M. Hentschel, R. Kienberger, M. Uiberacker, V. Yakovlev, A. Scrinzi, T. Westerwalbesloh, U. Kleineberg, U. Heinzmann, and F. Krausz, Nature (London) 419, 803 (2002).

[4] M. Jurvansuu, A. Kivimäki, and S. Aksela, Phys. Rev. A 64, 012502 (2001).
[5] J. M. Schins, P. Breger, P. Agostini, R. C. Constantinescu, H. G. Muller, G. Grillon, A. Antonetti, and A. Mysyrowicz, Phys. Rev. Lett. 73, 2180 (1994).

[6] M. Meyer et al., Phys. Rev. Lett. 108, 063007 (2012).

[7] T. Mazza, K. G. Papamihail, P. Radcliffe, W. B. Li, T. J. Kelly, J. T. Costello, S. Düsterer, P. Lambropoulos, and M. Meyer, J. Phys. B 45, 141001 (2012).

[8] P. Ranitovic, X. M. Tong, C. W. Hogle, X. Zhou, Y. Liu, N. Toshima, M. M. Murnane, and H. C. Kapteyn, Phys. Rev. Lett. 106, 053002 (2011).

[9] B. Cooper and V. Averbukh, Phys. Rev. Lett. 111, 083004 (2013).

[10] D. Iablonskyi et al., Phys. Rev. Lett. 119, 073203 (2017).

[11] M. Shapiro and P. Brumer, Adv. At. Mol. Opt. Phys. 42, 287 (2000).

[12] H. Stapelfeldt and T. Seideman, Rev. Mod. Phys. 75, 543 (2003).

[13] L. Giannessi, E. Allaria, K. C. Prince, C. Callegari, G. Sansone, K. Ueda, T. Morishita, C. N. Liu, A. N. GrumGrzhimailo, E. V. Gryzlova, N. Douguet, and K. Bartschat, Sci. Rep. 8, 7774 (2018).

[14] S. Anderson, D. Rider, and R. Zare, Chem. Phys. Lett. 93, 11 (1982).

[15] See Supplemental Material http://link.aps.org/supplemental/ 10.1103/PhysRevLett.122.233001 for more details, which includes Refs. [16-24].

[16] K. C. Prince et al., Nat. Photonics 10, 176 (2016).

[17] E. V. Gryzlova, A. N. Grum-Grzhimailo, E. I. Staroselskaya, N. Douguet, and K. Bartschat, Phys. Rev. A 97, 013420 (2018).

[18] S. Chatterjee and T. Nakajima, Phys. Rev. A 94, 023417 (2016).

[19] N. Rohringer and R. Santra, Phys. Rev. A 77, 053404 (2008).

[20] B. Cooper, P. Kolorenč, L. J. Frasinski, V. Averbukh, and J. P. Marangos, Faraday Discuss. 171, 93 (2014).

[21] P. W. Langhoff, Chem. Phys. Lett. 22, 60 (1973).

[22] P. W. Langhoff, C. T. Corcoran, J. S. Sims, F. Weinhold, and R. M. Glover, Phys. Rev. A 14, 1042 (1976).

[23] A. Cesar and H. Ågren, Phys. Rev. A 45, 2833 (1992).

[24] L. S. Cederbaum and F. Tarantelli, J. Chem. Phys. 99, 5871 (1993).

[25] M. V. Fedorov, Atomic and Free Electrons in a Strong Light Field (World Scientific, Singapore, 1997).

[26] M. V. Fedorov, J. Phys. B 10, 2573 (1977).

[27] M. E. Sukharev, E. Charron, A. Suzor-Weiner, and M. V. Fedorov, Int. J. Quantum Chem. 99, 452 (2004).

[28] M. Protopapas and P. L. Knight, J. Phys. B 28, 4459 (1995).

[29] A. B. Trofimov and J. Schirmer, J. Chem. Phys. 123, 144115 (2005).

[30] M. Ruberti, R. Yun, K. Gokhberg, S. Kopelke, L. S. Cederbaum, F. Tarantelli, and V. Averbukh, J. Chem. Phys. 139, 144107 (2013).

[31] M. Ruberti, R. Yun, K. Gokhberg, S. Kopelke, L. S. Cederbaum, F. Tarantelli, and V. Averbukh, J. Chem. Phys. 140, 184107 (2014).

[32] M. Ruberti, V. Averbukh, and P. Decleva, J. Chem. Phys. 141, 164126 (2014).

[33] C.-M. Liegener, Chem. Phys. Lett. 151, 83 (1988). 
[34] N. Kosugi, K. Ueda, Y. Shimizu, H. Chiba, M. Okunishi, K. Ohmori, Y. Sato, and E. Shigemasa, Chem. Phys. Lett. 246, 475 (1995).

[35] G. Prümper, V. Carravetta, Y. Muramatsu, Y. Tamenori, M. Kitajima, H. Tanaka, C. Makochekanwa, M. Hoshino, X. J. Liu, and K. Ueda, Phys. Rev. A 76, 052705 (2007).

[36] R. Feifel, J. H. D. Eland, R. J. Squibb, M. Mucke, S. Zagorodskikh, P. Linusson, F. Tarantelli, P. Kolorenč, and V. Averbukh, Phys. Rev. Lett. 116, 073001 (2016).
[37] F. Calegari, D. Ayuso, A. Trabattoni, L. Belshaw, S. D. Camillis, S. Anumula, F. Frassetto, L. Poletto, A. Palacios, P. Decleva, J. B. Greenwood, F. Martin, and M. Nisoli, Science 346, 336 (2014).

[38] P. M. Kraus, B. Mignolet, D. Baykusheva, A. Rupenyan, L. Horny, E. F. Penka, G. Grassi, O. I. Tolstikhin, J. Schneider, F. Jensen, L. B. Madsen, A. D. Bandrauk, F. Remacle, and H. J. Wörner, Science 350, 790 (2015). 